\title{
DOMAINS IN THE NONPERTURBATIVE QCD VACUUM
}

\author{
ALEXANDER C. KALLONIATIS* \\ CSSM, University of Adelaide, \\ Adelaide, 5005, South Australia, Australia \\ E-mail: akalloni@physics.adelaide.edu.au \\ SERGEI N. NEDELKO ${ }^{\dagger}$ \\ Bogoliubov L. of Theoretical Physics, JINR, \\ 141980 Dubna, Russian Federation \\ E-mail: nedelko@thsun1.jinr.ru
}

QCD vacuum gluon fields are modelled by hyperspherical domains of constant field strength. The pure glue theory confines static quarks. Solutions of the Dirac operator have chirality properties in agreement with lattice results.

Long-range gluon fields in the QCD vacuum are suspected to be sources for confinement and chiral-symmetry breaking. We proposed ${ }^{1}$ a Euclideanspace model for such fields as idealised domains of hyperspherical geometry enclosing regions of covariantly constant Abelian self-dual or anti-self-dual field strength ${ }^{2}$. The domains are characterised by two parameters, $R$ and $B$, the average domain size and field strength magnitudes respectively. We assign the following boundary conditions to gluon $A_{\mu}$ and quark $\psi$ fluctuations respectively at the $j$-th domain: $\breve{n}_{(j)} A_{\mu}^{(j)}=0$, and

$$
\psi=i \not \eta^{(j)} e^{i \alpha_{j} \gamma_{5}} \psi, \bar{\psi}=-\bar{\psi} i \not \eta^{(j)} e^{-i \alpha_{j} \gamma_{5}},
$$

for quarks, with antihermitian $\gamma$ matrices and $\gamma_{5}=\gamma_{1} \gamma_{2} \gamma_{3} \gamma_{4}$. These conditions can arise from the presence of finite action singular fields at the boundaries. The adjoint matrix $\breve{n}_{(j)}=T^{a} n_{(j)}^{a}, n_{(j)}^{a}$ a constant unit vector selecting the Cartan subalgebra, appears in the condition for gluons, and for the quarks the unit radial vector $\eta_{\mu}^{(j)}(x)=x_{\mu} /|x|$ is normal to the $j$-th surface. The covariantly constant (anti-)-self-dual mean field

\footnotetext{
* Supported by the Australian Research Council.
}

${ }^{\dagger}$ Partially supported by RFBR grant 01-02-17200. 
$F_{\mu \nu}^{a}(x)=\sum_{j=1}^{N} n^{(j) a} B_{\mu \nu}^{(j)} \theta\left(1-\left(x-z_{j}\right)^{2} / R^{2}\right)$, with $B_{\mu \nu}^{(j)} B_{\mu \rho}^{(j)}=B^{2} \delta_{\nu \rho}$ in each domain, compensates for a decoupled treatment of domains.

To zeroeth order in gluon fluctuations the model gives a gluon condensate density $g^{2}\left\langle F_{\mu \nu}^{a}(x) F_{\mu \nu}^{a}(x)\right\rangle=4 B^{2}$, topological charge per domain $q=$ $B^{2} R^{4} / 16$, topological susceptibility $\chi=B^{4} R^{4} / 128 \pi^{2}$, and an $S U(3)_{\text {colour }}$ string tension of the form $\sigma=B f\left(B R^{2}\right)$ with the function

$$
f(z)=\frac{2}{3 \pi z}\left(3-\frac{\sqrt{3}}{2 \pi z} \int_{0}^{2 \pi z / \sqrt{3}} \frac{d x}{x} \sin x-\frac{2 \sqrt{3}}{\pi z} \int_{0}^{\pi z / \sqrt{3}} \frac{d x}{x} \sin x\right)
$$

which is positive for $z>0$ and has a maximum for $z=1.55$. Estimating the model parameters at this $z$-value we fit the string constant to the lattice result, and obtain $\sqrt{B}=947 \mathrm{MeV}, R^{-1}=760 \mathrm{MeV}$, and thus get for the gluonic parameters of the vacuum $\sqrt{\sigma}=420 \mathrm{MeV}, \chi=(197 \mathrm{MeV})^{4}, \frac{\alpha_{s}}{\pi}\left\langle F^{2}\right\rangle=$ $0.081(\mathrm{GeV})^{4}$ and $q=0.15$, while the density of the system is $42 \mathrm{fm}^{-4}$. An area law does not occur for adjoint charges because of the presence of zero eigenvalues of the adjoint matrix $n^{a} T^{a}$.

The massless Dirac operator in a domain,

$$
(i \not D-\lambda) \psi(x)=0, D_{\mu}=\partial_{\mu}+\frac{i}{2} \hat{n} B_{\mu \nu} x_{\nu},
$$

subject to the boundary condition Eq. (1), is exactly soluble ${ }^{3}$. The matrix $\hat{n}$ is in the fundamental representation. We decompose eigenspinors via

$$
\psi_{\kappa}=i \not \chi \chi_{\kappa}+\varphi_{\kappa}, \gamma_{5} \chi_{\kappa}=\mp \chi_{\kappa}, \gamma_{5} \varphi_{\kappa}=\mp \varphi_{\kappa},
$$

where $\chi_{\kappa}=-\frac{1}{i \lambda_{\kappa}} \sqcap D \varphi_{\kappa}$, and $D^{2} \varphi_{\kappa}=\lambda_{\kappa}^{2} \varphi_{\kappa}$. To give the quantum numbers $\kappa$ we use projectors $N_{ \pm}=\frac{1}{2}(1 \pm \hat{n} /|\hat{n}|)$ and $\Sigma_{ \pm}=\frac{1}{2}(1 \pm \vec{\Sigma} \vec{B} / B)$ with $\hat{B}=|\hat{n}| B$ and the mixed projector $O_{\zeta}=N_{+} \Sigma_{\zeta}+N_{-} \Sigma_{-\zeta}$ with $\zeta= \pm$. Then $\kappa=\left(\zeta, n, k, m_{1,2}\right)$. Eq.(1) gives $\chi_{\kappa}=e^{\mp i \alpha} \varphi_{\kappa}$ on the boundary if the domain is (anti-)self-dual, possible only if the chiralities of the spinors $\chi$ and $\varphi$ are consistent with the duality of the vacuum gluon field: $\gamma_{5} \varphi=\mp \varphi$ and $\gamma_{5} \chi=\mp \chi$ if the field $B_{\mu \nu}$ is (anti-)self-dual. The discrete eigenvalues $\lambda_{\kappa}$ include then a principal quantum number $n=1,2, \ldots$. The angular momentum orbital and azimuthal quantum numbers take values $k=0,1, \ldots$ and $m_{1,2}=-k / 2, \ldots, k / 2$. Zero modes $\lambda=0$ are absent.

As an application, we study the local chirality parameter $X(x)$ defined $^{4}$ via $\tan \left(\frac{\pi}{4}(1-X(x))\right)=\sqrt{\frac{\psi^{\dagger}(x)\left(1-\gamma_{5}\right) \psi(x)}{\psi^{\dagger}(x)\left(1+\gamma_{5}\right) \psi(x)}}$ which gives $X(x)= \pm 1$ at $x$ where $\gamma_{5} \psi(x)= \pm \psi(x)$. For lattice overlap fermions, histograms of $X$ measured at maxima of $\psi^{\dagger} \psi$ for low-lying overlap-Dirac eigenmodes peak ${ }^{5}$ at $X \approx \pm 1$ suggesting that low-lying modes are strongly chiral and filter the 
duality of "lumps" underlying the gauge field fluctuations. At domain centres radial modes are exactly chiral and probability densities are maximal. The "width" of the peaks at half-maximum for these modes is $\alpha$-dependent and of the order $0.12-0.14 \mathrm{fm}$. The chirality of the modes are characterised via a histogram by averaging $X(x)$ over a small neighborhood of the domain centre. With all $\alpha$ equally probable, we compute the probability to find a given value of smeared $X$ among a set of modes. The result in Fig. 1 is for the lowest modes and all spin-color orientations. The double peaks at $X \approx \pm 0.8$ and the above values for width and density of domains are consistent with the lattice ${ }^{5,6}$. This is argued to signal spontaneously broken chiral symmetry on the lattice. In our model this is achieved without fermionic zero modes; strongly chiral low-lying modes suffice. Explicit calculation of the chiral condensate in the model is underway, though an estimate of the condensate density at the domain centre ${ }^{1}$ gives $-(228 \mathrm{MeV})^{3}$.

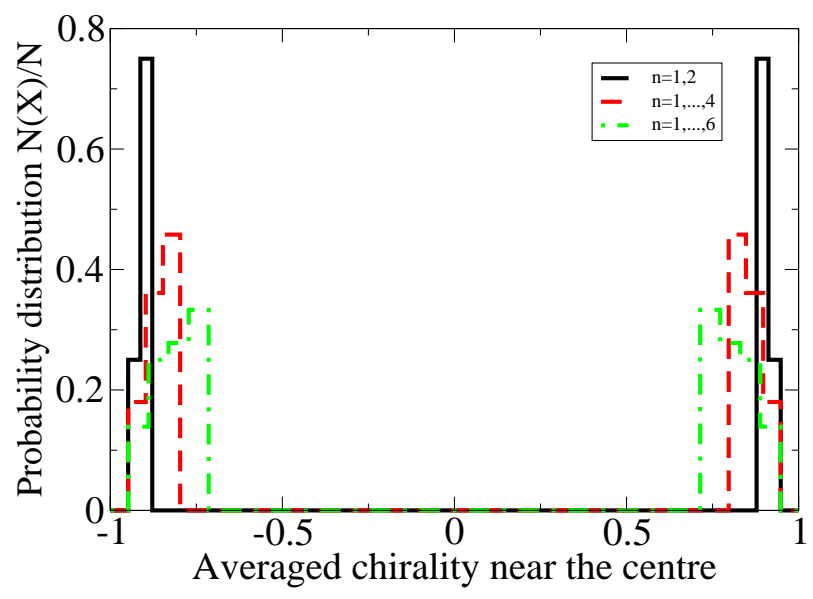

Figure 1. Histogram of chirality parameter $\bar{X}$ averaged over the central region with radius $0.025 \mathrm{fm}$. Plots given in solid, dashed and dot-dashed lines incorporate all modes with $n \leq 2, n \leq 4$ and $n \leq 6$ respectively.

\section{References}

1. A.C. Kalloniatis, S.N. Nedelko, Phys.Rev. D64, 114025 (2001).

2. H. Leutwyler, Nucl.Phys. B179, 129 (1980).

3. A.C. Kalloniatis, S.N. Nedelko, Phys.Rev. D66 074020 (2002).

4. I. Horvath et al., Phys.Rev. D65, 014502 (2002).

5. R.G. Edwards, U.M. Heller, Phys.Rev. D65, 014505 (2002).

6. C. Gattringer et al., Nucl.Phys. B618, 205 (2001); C. Gattringer et al., ibid B617, 101 (2001); I. Horvath et al., Phys.Rev. D66, 034501 (2002). 